\title{
Ortho-para transition in molecular hydrogen
}

\author{
Krzysztof Pachucki* \\ Institute of Theoretical Physics, University of Warsaw, Hoża 69, 00-681 Warsaw, Poland \\ Jacek Komasa ${ }^{\dagger}$ \\ Faculty of Chemistry, A. Mickiewicz University, Grunwaldzka 6, 60-780 Poznań, Poland
}

(Received 22 December 2007; published 14 March 2008)

\begin{abstract}
The radiative ortho-para transition in molecular hydrogen is studied. This highly forbidden transition is very sensitive to relativistic and subtle nonadiabatic effects. Our result for the transition rate in the ground vibrational level $\Gamma(J=1 \rightarrow J=0)=6.20(62) \times 10^{-14} \mathrm{yr}^{-1}$ is significantly lower in comparison to all the previous approximate calculations. Experimental detection of such a weak line by observation of, for example, cold interstellar molecular hydrogen is at present unlikely.
\end{abstract}

DOI: 10.1103/PhysRevA.77.030501

The hydrogen molecule in the ground electronic state can exist in a nuclear triplet state $\left(S=1\right.$, ortho- $\left.\mathrm{H}_{2}\right)$ with the odd angular momentum $L$, or in a singlet state $\left(S=0\right.$, para- $\left.\mathrm{H}_{2}\right)$ with the even $L$. The question we raise is, what are the physical mechanisms for possible transitions between these two classes of states? The nonradiative transition, for example, in interstellar molecular hydrogen is mostly induced by collisions with atomic $\mathrm{H}$. The corresponding rates were obtained by Sun and Dalgarno in [1]. The radiative transition which is much weaker can, in principle, take place at sufficiently low densities and temperatures. The relativistic spin-orbit interaction (nuclear spin and the electron momenta) is the most obvious source of this transition as it mixes slightly the ortho- $\mathrm{H}_{2}$ and para- $\mathrm{H}_{2}$ states [see Eq. (1)]. This effect has been considered in the original work of Raich and Good in [2], although not in a complete and systematic way. It has happened that a tiny nonadiabatic correction to the total $\mathrm{H}_{2}$ wave function significantly changes the theoretical predictions for this rate. Moreover, the spin-orbit mixing is not the only effect, which makes this transition possible. There are also relativistic corrections to the $E 1$ coupling to the electromagnetic field which are barely known in the literature. These corrections in the context of the $\mathrm{H}_{2}$ molecule have been derived for the first time by Dodelson in [3] using the Feinberg-Sucher formalism. Here we rederive this result in a much simpler way. Because of the summation over the infinite $\mathrm{H}_{2}$ spectrum, the calculations of the ortho-para transition amplitude are not completely trivial. The most elaborate calculations so far, by Raich and Good [2] including Dodelson corrections [3], gave the rate of 1.85(46) $\times 10^{-13} \mathrm{yr}^{-1}$, which did not include all important contributions. The purpose of this work is to present a complete theoretical description of the radiative ortho-para conversion of the $\mathrm{H}_{2}$ molecule, including the results of direct numerical calculations of the transition probability for the lowest rotational levels.

The interaction of an arbitrary molecule with the electromagnetic field, whose characteristic wavelength is much larger than the size of this molecule, including the relevant spin-orbit interaction is [4]

\footnotetext{
*krp@fuw.edu.pl

†komasa@man.poznan.pl
}

PACS number(s): 31.15.ac, 31.30.J-, 33.70.Ca, 95.30.Ky

In the above, $A, b$ are the indices of the nuclei and electrons, respectively, $x_{A}, x_{b}$ are the coordinates of the nuclei and electrons with respect to the mass center, $\vec{x}_{A b}=\vec{x}_{A}-\vec{x}_{b}$, and $g_{A}$ is the nuclear $g$ factor. Moreover, the electromagnetic fields $\vec{E}, \vec{B}$ and their derivatives are assumed in Eq. (1) to be at the mass center.

For ortho- $\mathrm{H}_{2}$ (the first excited rotational state) the nuclear spin $S=1$ couples to the orbital angular momentum $J=1$ of the nuclei, giving the total angular momentum characterized by quantum numbers $F=0,1,2$. In the para- $\mathrm{H}_{2}$ (the ground rotational state) the total angular momentum is $F=0$, therefore the one photon ortho-para transition from any other $F$ $=0$ level is strictly forbidden, while the $F=1$ level decays by the $E 1$ transition and the $F=2$ level decays by the $M 2$ transition.

Let us first consider the $M 2$ transition from the $F=2$ level of ortho- $\mathrm{H}_{2}$, to the $F=0$ level of para- $\mathrm{H}_{2}$. This transition comes from the following interaction with the electromagnetic field, which is obtained from Eq. (1):

$$
\delta H=-\frac{e g_{p}}{4 m_{p}}\left(s_{A}^{j}-s_{B}^{j}\right) \mathcal{R}^{i} B_{, i}^{j},
$$

with $\mathcal{R}=x_{A}-x_{B}$ and the proton $g$ factor $g_{p}$ $=5.585694713$ (46) [5]. From this Hamiltonian one obtains the transition rate $\Gamma(M 2) \equiv \Gamma_{2}$, 


$$
\begin{aligned}
\Gamma_{2} & =2 \alpha \omega \frac{1}{5} \sum_{m_{F}}\left|\left\langle{ }^{1} \Sigma_{0}\left|\frac{g_{p}}{4 m_{p}}(\vec{k} \cdot \overrightarrow{\mathcal{R}})\left(\vec{s}_{A}-\vec{s}_{B}\right) \times \vec{k}\right|{ }^{3} \Pi_{2}, m_{F}\right\rangle\right|^{2} \\
& \approx \frac{1}{120} \alpha \omega^{5}\left(\frac{g_{p} \mathcal{R}_{0}}{m_{p}}\right)^{2}
\end{aligned}
$$

where $\mathcal{R}_{0}$ is the average distance between protons, and the last equation holds for the nuclear $\mathrm{H}_{2}$ wave function, which is strongly peaked around $\mathcal{R}_{0}$, as is the case for the lowest rovibrational levels.

The calculation of the rate for the $E 1$ transition from the $F=1$ level of ortho- $\mathrm{H}_{2}$ to the $F=0$ level of para- $\mathrm{H}_{2}$ is more complicated as it includes corrections to the wave function coming from the spin-orbit interaction. Since it is the $\Delta F$ $=1$ transition, the operators in the interaction Hamiltonian in Eq. (1) can be simplified, namely, $x_{A}^{i} s_{A}^{j} \rightarrow \epsilon^{i j k}\left(\vec{x}_{A} \times \vec{s}_{A}\right)^{k} / 2$ and this Hamiltonian becomes

$$
\begin{gathered}
\delta H=H_{L S}+e\left(\vec{x}_{1 C}+\vec{x}_{2 C}\right) \cdot \vec{E} \\
+\frac{e}{4 m_{p}}\left(\frac{g_{p}}{2}-1\right) \overrightarrow{\mathcal{R}} \times\left(\vec{s}_{A}-\vec{s}_{B}\right) \cdot \partial_{t} \vec{E}, \\
H_{L S}=-\frac{i}{2} \vec{H}_{L S} \cdot\left(\vec{s}_{A}-\vec{s}_{B}\right), \\
\vec{H}_{L S}=\frac{g_{p} \alpha}{2 m_{p} m} \vec{h}_{1}-\frac{\left(g_{p}-1\right) \alpha}{2 m_{p}^{2}} \vec{h}_{2} \times \vec{\nabla}_{\mathcal{R}}, \\
\vec{h}_{1}=\left(\frac{\vec{x}_{1 A}}{x_{1 A}^{3}}-\frac{\vec{x}_{1 B}}{x_{1 B}^{3}}\right) \times \vec{\nabla}_{1}+\left(\frac{\vec{x}_{2 A}}{x_{2 A}^{3}}-\frac{\vec{x}_{2 B}}{x_{2 B}^{3}}\right) \times \vec{\nabla}_{2}, \\
\vec{h}_{2}=\frac{\vec{x}_{1 A}}{x_{1 A}^{3}}+\frac{\vec{x}_{1 B}}{x_{1 B}^{3}}+\frac{\vec{x}_{2 A}}{x_{2 A}^{3}}+\frac{\vec{x}_{2 B}}{x_{2 B}^{3}} .
\end{gathered}
$$

The resulting transition rate $\Gamma(E 1) \equiv \Gamma_{1}$ is

$$
\begin{aligned}
\Gamma_{1} & =\frac{4}{3} \alpha \omega \frac{1}{3} \sum_{m_{F}}\left|\left\langle{ }^{1} \Sigma_{0}\left|\vec{Q} \times \frac{\left(\vec{s}_{A}-\vec{s}_{B}\right)}{2}\right|{ }^{3} \Pi_{1}, m_{F}\right\rangle\right|^{2} \\
& =\frac{2}{9} \alpha \omega\left|\left\langle\Sigma\left|Q^{k}\right| \Pi^{k}\right\rangle\right|^{2},
\end{aligned}
$$

where

$$
\begin{aligned}
Q^{k}= & \frac{1}{2 m_{p}}\left(\frac{g_{p}}{2}-1\right) \omega^{2} \mathcal{R}^{k}-\frac{\omega}{2} \epsilon^{i k j}\left(x_{1 C}^{i}+x_{2 C}^{i}\right) \frac{1}{E_{\Pi}-H} H_{L S}^{j} \\
& -\frac{\omega}{2} \epsilon^{i k j} H_{L S}^{j} \frac{1}{E_{\Sigma}-H}\left(x_{1 C}^{i}+x_{2 C}^{i}\right),
\end{aligned}
$$

and $H$ is the four-body nonrelativistic Hamiltonian. In order to simplify the evaluation of $Q^{k}$ we perform the adiabatic expansion, namely, the expansion of the resolvent in the kinetic energy of the nuclei

$$
\begin{aligned}
\frac{1}{E_{\Pi}-H}= & \frac{1}{E_{B O}-H_{B O}}-\frac{1}{E_{B O}-H_{B O}}\left(\delta E_{\Pi}-\delta H_{M}\right) \\
& \times \frac{1}{E_{B O}-H_{B O}}+\cdots,
\end{aligned}
$$

similarly for $\frac{1}{E_{\Sigma}-H}$, and the expansion of the wave function

$$
\begin{aligned}
& \phi_{\Sigma}=\psi\left(\vec{x}_{1 C}, \vec{x}_{2 C} ; \mathcal{R}\right) \lambda_{0}(\mathcal{R}) / \sqrt{4 \pi}+\delta \phi_{\Sigma}, \\
& \phi_{\Pi}^{k}=\psi\left(\vec{x}_{1 C}, \vec{x}_{2 C} ; \mathcal{R}\right) \lambda_{1}^{k}(\overrightarrow{\mathcal{R}}) / \sqrt{4 \pi}+\delta \phi_{\Pi},
\end{aligned}
$$

with $\lambda_{1}^{k}=\lambda_{1} \mathcal{R}^{k} / \mathcal{R}$ and with normalization

$$
\int d \mathcal{R} \mathcal{R}^{2} \lambda_{0}^{2}(\mathcal{R})=\int d \mathcal{R} \mathcal{R}^{2} \lambda_{1}^{2}(\mathcal{R})=1
$$

While the exact nonadiabatic correction to the wave function is unknown, we need only the first order $m / m_{p}$ part of the correction, which explicitly depends on the nuclear state [6]

$$
\begin{aligned}
& \delta \phi_{\Sigma}=-\frac{2}{m_{p}} \frac{1}{E_{B O}-H_{B O}} \nabla_{\mathcal{R}}^{l} \psi \nabla^{l} \lambda_{0} / \sqrt{4 \pi}, \\
& \delta \phi_{\Pi}^{k}=-\frac{2}{m_{p}} \frac{1}{E_{B O}-H_{B O}} \nabla_{\mathcal{R}}^{l} \psi \nabla^{l} \lambda_{1}^{k} / \sqrt{4 \pi} .
\end{aligned}
$$

We introduce now the perturbed electronic wave functions

$$
\begin{gathered}
\psi_{1}^{i}=\frac{1}{E_{B O}-H_{B O}}\left(x_{1 C}^{i}+x_{2 C}^{i}\right) \psi, \\
\psi_{2}^{j}=\frac{1}{E_{B O}-H_{B O}} h_{1}^{j} \psi, \\
\psi_{3}^{l}=\frac{1}{E_{B O}-H_{B O}} \nabla_{\mathcal{R}}^{l} \psi,
\end{gathered}
$$

to simplify the matrix elements of $Q^{k}$ in Eq. (10),

$$
\begin{aligned}
\left\langle\Sigma\left|Q^{k}\right| \Pi^{k}\right\rangle= & \frac{1}{2 m_{p}}\left(\frac{g_{p}}{2}-1\right) \omega^{2} \mathcal{R}_{0}-\frac{\omega\left(g_{p}-1\right)}{m_{p}^{2} \mathcal{R}_{0}} \\
& +\omega^{2} \frac{g_{p} \alpha}{4 m_{p} m} X_{1}-\omega \frac{g_{p} \alpha}{4 m_{p}^{2} m \mathcal{R}_{0}}\left(X_{2}+2 X_{3}\right),
\end{aligned}
$$

where we used the commutator

$$
i\left[p_{1}^{k}+p_{2}^{k}, H_{B O}-E_{B O}\right]=\alpha h_{2}^{k},
$$

and

$$
\begin{gathered}
X_{1}=\epsilon^{i k j} n^{k}\left\langle\psi_{1}^{i} \mid \psi_{2}^{j}\right\rangle_{\mathcal{R}_{0}}, \\
X_{2}=\left(\delta^{k l}-n^{k} n^{l}\right) \epsilon^{i k j}\left[\left\langle\partial_{\mathcal{R}}^{l} \psi_{1}^{i} \mid \psi_{2}^{j}\right\rangle-\left\langle\psi_{1}^{i} \mid \partial_{\mathcal{R}}^{l} \psi_{2}^{j}\right\rangle\right]_{\mathcal{R}_{0}},
\end{gathered}
$$




$$
X_{3}=\left(\delta^{k l}-n^{k} n^{l}\right) \epsilon^{i k j}\left[\left\langle\psi_{3}^{l}\left|\left(x_{1 C}^{i}+x_{2 C}^{i}\right)\right| \psi_{2}^{j}\right\rangle+\left\langle\psi_{3}^{l}\left|h_{1}^{j}\right| \psi_{1}^{i}\right\rangle\right]_{\mathcal{R}_{0}},
$$

with $\vec{n} \equiv \overrightarrow{\mathcal{R}}_{0} / \mathcal{R}_{0}$. One notes that derivatives of nonlinear and linear parameters in $\psi$ [see Eq. (24)] with respect to $\mathcal{R}$ do not contribute to the above matrix elements, which significantly simplifies the numerical computations.

Results for $X_{i}$ can be expressed in terms of dimensionless factors $F_{i}$,

$$
\begin{aligned}
& X_{1}=-\frac{9}{m^{2} \alpha^{4} \mathcal{R}^{2}} F_{1}(m \alpha \mathcal{R}), \\
& X_{2}=\frac{9}{m^{2} \alpha^{4} \mathcal{R}^{3}} F_{2}(m \alpha \mathcal{R}), \\
& X_{3}=-\frac{2 m}{\alpha} F_{3}(m \alpha \mathcal{R}),
\end{aligned}
$$

which are chosen in such a way that $F_{i}(m \alpha \mathcal{R})$ vanish at $\mathcal{R}$ $=0$ and approach 1 for $\mathcal{R} \rightarrow \infty$. However, in the case of $F_{2}$ this large $\mathcal{R}$ limit is only a rough approximation, since we have not been able to perform this limit analytically. We can now return to Eq. (18) and obtain a compact formula for the matrix element in the transition rate $\Gamma_{1}$ of Eq. (9),

$$
\begin{aligned}
\left\langle\Sigma\left|Q^{k}\right| \Pi^{k}\right\rangle= & \frac{\omega^{2} \mathcal{R}_{0}}{2 m_{p}}\left[\left(\frac{g_{p}}{2}-1\right)-\frac{9}{2} \frac{g_{p} F_{1}}{\left(m \alpha \mathcal{R}_{0}\right)^{3}}\right] \\
& -\frac{\omega}{m_{p}^{2} \mathcal{R}_{0}}\left[\left(g_{p}-1\right)-g_{p} F_{3}+\frac{9}{4} \frac{g_{p} F_{2}}{\left(m \alpha \mathcal{R}_{0}\right)^{3}}\right] .
\end{aligned}
$$

Numerical evaluation of the $\Gamma_{2}$ rate according to formula (3) is straightforward. To obtain the ortho-para energy spacing $\omega$ and the average internuclear distance $\mathcal{R}_{0}$, we employed the accurate Kołos-Le Roy-Schwartz interaction potential [7], which includes the adiabatic and relativistic energy corrections. With this potential we solved numerically the radial Schrödinger equation to obtain the energies and wave functions corresponding to the lowest ortho and para levels. The numerical values used here are $\omega=2 \pi$ $\times 118.49 \mathrm{~cm}^{-1}$ and $\mathcal{R}_{0}=1.449$ a.u., and the resulting $M 2$ transition rate with physical constants from Ref. [5] is

$$
\Gamma_{2}=1.07(1) \times 10^{-14} \mathrm{yr}^{-1} .
$$

The accurate evaluation of $\Gamma_{1}$ and the corresponding electronic matrix elements $F_{i}$ in Eqs. (21) is a challenging task. We have represented the electronic ground state wave function as well as the first order perturbed functions defined by Eqs. (17), in the form of properly symmetrized linear combinations $\psi=\sum_{k} c_{k} \hat{P}_{g, u} \phi_{k}$ of Gaussian geminals

$$
\phi_{k}=\Xi_{k} \exp \left(-\alpha_{k} x_{1 A}^{2}-\beta_{k} x_{1 B}^{2}-\zeta_{k} x_{2 A}^{2}-\eta_{k} x_{2 B}^{2}-\gamma_{k} x_{12}^{2}\right) .
$$

The projection operators
TABLE I. The definitions of the functions used in the computations.

\begin{tabular}{lccc}
\hline \hline & $\Xi_{k}$ & $\hat{P}$, Eq. (25) & $\hat{\mathcal{O}}$, Eq. (26) \\
\hline$\psi$ & 1 & Gerade & \\
$\psi_{1}^{x}$ & $x_{1}, x_{2}$ & Ungerade & $x_{1}+x_{2}$ \\
$\psi_{1}^{y}$ & $y_{1}, y_{2}$ & Ungerade & $y_{1}+y_{2}$ \\
$\psi_{1}^{z}$ & $z_{1}, z_{2}$ & Ungerade & $z_{1}+z_{2}$ \\
$\psi_{2}^{x}$ & $y_{1} z_{2}-y_{2} z_{1}$ & Ungerade & $h_{1}^{x}$ \\
$\psi_{2}^{z}$ & $y_{1}, y_{2}$ & Ungerade & $h_{1}^{z}$ \\
$\psi_{3}^{y}$ & $y_{1}, y_{2}$ & Gerade & $\partial_{\mathcal{R}}^{y}$ \\
\hline \hline
\end{tabular}

$$
\hat{P}_{g, u}=\frac{1}{4}\left(1+\hat{P}_{12}\right)(1 \pm \hat{1})
$$

ensure the proper symmetry with respect to the exchange of the electrons and with respect to the inversion operation, yielding singlet gerade or ungerade functions. Required $\Sigma^{+}$, $\Sigma^{-}$, or $\Pi$ symmetry of the electronic wave function was imposed by the Cartesian prefactor $\Xi_{k}$. The linear and the nonlinear parameters were optimized variationally with the goal function being the ground state energy in the case of the unperturbed wave function $\psi$ or pertinent Hylleraas functional

$$
\mathcal{J}\left[\psi_{k}^{i}\right]=\left\langle\psi_{k}^{i}\left|E_{B O}-H_{B O}\right| \psi_{k}^{i}\right\rangle+2\left\langle\psi|\hat{\mathcal{O}}| \psi_{k}^{i}\right\rangle,
$$

in the case of the perturbed functions $\psi_{k}^{i}$. Table I shows ex-

TABLE II. Numerical values of the optimum goal functions Eq. (26) and the expectation values comprising the $X_{i}$ factors Eqs. (27) in atomic units.

\begin{tabular}{lccc}
\hline \hline & $\mathcal{R}_{0}=1.449$ & $\mathcal{R}=12.0$ & Asymptotic \\
\hline$E_{B O}$ & -1.174073569 & -1.000002546 & -1.0 \\
$\mathcal{J}\left[\psi_{1}^{x}\right]$ & -3.3582 & -4.5241 & -4.5 \\
$\mathcal{J}\left[\psi_{1}^{y}\right]$ & -2.3684 & -4.4885 & -4.5 \\
$\mathcal{J}\left[\psi_{2}^{x}\right]$ & $-7.87 \times 10^{-3}$ & $-2.22 \times 10^{-7}$ & 0.0 \\
$\mathcal{J}\left[\psi_{2}^{z}\right]$ & -0.4925 & $-5.06 \times 10^{-5}$ & 0.0 \\
$\mathcal{J}\left[\psi_{3}^{y}\right]$ & $-2.90 \times 10^{-2}$ & -0.2500 & -0.25 \\
$\left\langle\psi_{1}^{y} \mid \psi_{2}^{z}\right\rangle$ & 0.7824 & 0.0312 & \\
$X_{1}$ & -1.5649 & -0.0623 & \\
$F_{1}$ & 0.3651 & 0.9975 & 1.0 \\
$\left\langle\partial_{\mathcal{R}}^{y} \psi_{1}^{x} \mid \psi_{2}^{z}\right\rangle$ & 0.7467 & 0.0014 & \\
$\left\langle\psi_{1}^{z} \mid \partial_{\mathcal{R}}^{y} \psi_{2}^{x}\right\rangle$ & 0.0034 & 0.0002 & \\
$X_{2}$ & 3.0003 & 0.0062 & \\
$F_{2}$ & 1.0142 & 1.1989 & \\
$\left\langle\psi_{3}^{y}\left|\left(x_{1 C}^{x}+x_{2 C}^{x}\right)\right| \psi_{2}^{z}\right\rangle$ & 0.0707 & -0.0029 & \\
$\left\langle\psi_{3}^{y}\left|\left(x_{1 C}^{z}+x_{2 C}^{z}\right)\right| \psi_{2}^{x}\right\rangle$ & -0.0010 & -0.0001 & \\
$\left\langle\psi_{3}^{y}\left|h_{1}^{z}\right| \psi_{1}^{x}\right\rangle$ & -0.2579 & -0.5026 & \\
$\left\langle\psi_{3}^{y}\left|h_{1}^{x}\right| \psi_{1}^{z}\right\rangle$ & 0.1066 & 0.4980 & \\
$X_{3}$ & -0.3984 & -2.0037 & \\
$F_{3}$ & 0.1992 & 1.0019 & \\
\hline \hline
\end{tabular}


plicitly the elements defining particular functions with the assumption that the molecule is placed along the Cartesian $X$ axis. The unperturbed wave function has been expanded in a 600 -term basis set which enables the electronic ground state energy to be obtained with an error of only $3 \times 10^{-9}$ a.u. A 1200-term expansion has been employed to represent the perturbed functions. Values of the $\mathcal{J}$ functionals corresponding to the optimum parameters are displayed in Table II.

The general formulas (20), in the particular case of the molecule oriented along the $X$ axis, can be explicitly written as follows:

$$
\begin{aligned}
X_{1}= & -2\left\langle\psi_{1}^{y} \mid \psi_{2}^{z}\right\rangle_{\mathcal{R}_{0}}, \\
X_{2}= & 4\left[\left\langle\partial_{\mathcal{R}}^{y} \psi_{1}^{x} \mid \psi_{2}^{z}\right\rangle-\left\langle\partial_{\mathcal{R}}^{y} \psi_{1}^{z} \mid \psi_{2}^{x}\right\rangle\right]_{\mathcal{R}_{0}}, \\
X_{3}= & 2\left[\left\langle\psi_{3}^{y}\left|\left(x_{1 C}^{x}+x_{2 C}^{x}\right)\right| \psi_{2}^{z}\right\rangle-\left\langle\psi_{3}^{y}\left|\left(x_{1 C}^{z}+x_{2 C}^{z}\right)\right| \psi_{2}^{x}\right\rangle\right. \\
& \left.+\left\langle\psi_{3}^{y}\left|h_{1}^{z}\right| \psi_{1}^{x}\right\rangle-\left\langle\psi_{3}^{y}\left|h_{1}^{x}\right| \psi_{1}^{z}\right\rangle\right]_{\mathcal{R}_{0}} .
\end{aligned}
$$

Table II contains all the expectation values appearing in Eqs. (27) as well as the final $X_{i}$ and $F_{i}$ values computed at $\mathcal{R}_{0}$ $=1.449$ bohr. To check the correctness of our codes we performed additional calculations at large internuclear distance $(\mathcal{R}=12.0$ bohr) and compared the resulting goal functions and the expectation values with analytically derived asymptotic values. This comparison is presented in Table II.

Using Eqs. (9), (22), and Table II one obtains the numerical value for the $E 1$ transition rate

$$
\Gamma_{1}=1.68(17) \times 10^{-13} \mathrm{yr}^{-1},
$$

and finally the rate averaged over the total angular momentum $F$,

$$
\Gamma=\left(5 \Gamma_{2}+3 \Gamma_{1}\right) / 9=6.20(62) \times 10^{-14} \mathrm{yr}^{-1} .
$$

Our result for the averaged transition rate is in disagreement with the result of Dodelson [3], $\Gamma=1.85(46)$ $\times 10^{-13} \mathrm{yr}^{-1}$, which is in turn based on the former work of Raich and Good [2] and included direct coupling of nuclear spin to the radiation field. We confirm in this work the existence of these additional couplings, which here are expressed by the third term in Eq. (1). In our opinion, the difference from our result is due to the omission of the $M 2$ transition, omission of the nonadiabatic contributions corresponding to $X_{2}$ and $X_{3}$ in Eq. (20), less accurate $\omega$, and a lower accuracy of the numerical calculation of the matrix elements in Ref. [2]. In particular, without $X_{3}$ the overall rate $\Gamma$ would be about $24 \%$ larger.

The possibility of the experimental detection of the orthopara $\mathrm{H}_{2}$ line is questionable. Much stronger E2 lines have already been observed at the Infrared Space Observatory (ISO) and served for estimation of the temperature of interstellar hydrogen clouds and of the ratio of abundance ortho- $\mathrm{H}_{2}$ to para- $\mathrm{H}_{2}$, which sometimes differs significantly from the equilibrium one [8]. The much weaker $E 1$ line has not been observed yet. In fact there is a potential opportunity related with the Herschel Space Observatory to be launched in 2008 [9]. Its spectral range covers the ortho-para line at $84.4 \mu \mathrm{m}$, but its resolution is, probably, not high enough at this wavelength.

K.P. wishes to acknowledge interesting discussions with Krzysztof Meissner, and thanks the Laboratoire Kastler Brossel in Paris for kind hospitality during his stay, when this work was written.
[1] Y. Sun and A. Dalgarno, Astrophys. J. 427, 1053 (1994).

[2] J. C. Raich and R. H. Good, Jr., Astrophys. J. 139, 1004 (1964).

[3] S. Dodelson, J. Phys. B 19, 2871 (1986).

[4] K. Pachucki, Phys. Rev. A 76, 022106 (2007).

[5] P. J. Mohr and B. N. Taylor, Rev. Mod. Phys. 77, 1 (2005).
[6] K. Pachucki and J. Komasa, e-print arXiv:0801.0561.

[7] C. Schwartz and R. J. Le Roy, J. Mol. Spectrosc. 121, 420 (1987).

[8] D. A. Neufeld, G. J. Melnick, and M. Harwit, Astrophys. J. 506, L75 (1998).

[9] http://herschel.esac.esa.int 